Lilia José Corzo*

María Belén Guercio"

Hernán Pedro Vigier"

Recibido: 18 de agosto de 2020

Evaluación: 31 de marzo de 2021

Aprobado: 30 de julio de 2021

Artículo de investigación

(C) 2021 Universidad Católica de Colombia. Facultad de Ciencias

Económicas y Administrativas.

Todos los derechos reservados

* Docente y becaria, Licenciada en Economía (Universidad Nacional del Sur). Centro de Emprendedorismo y Desarrollo

Territorial Sostenible, Comisión de Investigaciones Científicas de la Provincia de Buenos Aires, Universidad Provincial del Sudoeste, Bahía Blanca, Buenos Aires,

Argentina. lilia.corzo@upso.edu.ar.

(D https://orcid.org/0000-0001-9133-6949

** Investigadora y docente, Doctora en Organización Industrial, Empresas

y Mercados (Universidad Rovira i

Virgili). Instituto de Investigaciones

Económicas y Sociales del Sur y

Universidad Provincial del Sudoeste,

Bahía Blanca, Buenos Aires, Argentina.

mbguercio@iiess-conicet.gob.ar.

(D https://orcid.org/0000-0001-5780-2854

\section{Alcance de los créditos a tasa subsidiada para mipymes Argentinas}

\section{Resumen}

El objetivo de este artículo es analizar el alcance de los créditos bancarios a tasa subsidiada para mipymes. Se estima un modelo Logit para examinar los determinantes de la demanda efectiva y potencial para empresas argentinas en 2017. Los principales resultados muestran que las empresas con necesidades insatisfechas de crédito bancario tradicional presentan una menor demanda efectiva y potencial. Además, se observa una relación positiva entre el tamaño de la empresa y la demanda efectiva. La demanda potencial, por su parte, es explicada por la forma jurídica, el acceso al crédito comercial, la educación del gerente y haber experimentado una pérdida de inversión por falta de crédito. Este estudio genera bases para rediseñar y mejorar el alcance de los programas de financiamiento para mipymes.

Palabras clave: acceso al crédito, Argentina, créditos a tasa subsidiada, demanda potencial, mipyme, políticas públicas.

JEL Classification: G28, H81 
*** Docente e investigador, doctor en Administración y Gestión de Empresas (Universidad Rovira i Virgili). Centro de Emprendedorismo y Desarrollo Territorial Sostenible, Comisión de Investigaciones Científicas de la Provincia de Buenos Aires, Universidad Provincial del Sudoeste y Departamento de Economía, Universidad Nacional del Sur, Bahía Blanca, Buenos Aires, Argentina. hvigier@upso.edu.ar. (D https://orcid.org/0000-0003-0774-8620

\section{Analysis of Argentine SMEs Subsidized Credit Scope}

\begin{abstract}
The aim of this research is to analyze the scope of subsidized bank credit for SMEs. A Logit model that examines the determinants of effective and potential demand is estimated for Argentine SMEs in 2017. Our main results show that firms excluded from traditional bank credit in the past present a lower potential and effective demand for subsidized credits. Moreover, we find a positive relationship between firm's size and effective demand. Besides, potential demand is explained by the legal status, the manager's level of education, access to trade credit and experienced a lost investment due to lack of credit. The novelty approach of this research lays knowledge to redesign the public financial support programs for SMEs and improve their scope.
\end{abstract}

Keywords: subsidized credit, access to credit, public policy, potential demand, Argentine, small and medium enterprises, firm. 


\section{INTRODUCCIÓN}

Las dificultades de las micro, pequeñas y medianas empresas (mipymes) para financiarse con fuentes externas están ampliamente argumentadas en la literatura académica (Beck y Demirguguc-Kunt, 2006). Dentro de las principales causas de dichas limitaciones, se encuentran los problemas de información asimétrica entre prestamistas y prestatarios (Stiglitz y Weiss, 1981), lo que tiene como consecuencia la exclusión del mercado de potenciales demandantes de fondos, así como los elevados costos de financiamiento soportados por las mipymes que acceden (Cole et al., 2004; de la Torre et al., 2010).

Dadas las particularidades en la estructura de capital de las mipymes y los problemas informacionales presentes en los mercados de crédito, este segmento de empresas es objeto de la intervención pública con el fin de mejorar su acceso al crédito (Berger y Udell, 2006; Beck y Demirguc-Kunt, 2006; de la Torre et al., 2010; Beck et al., 2011; Heredia y Sánchez, 2016; Briozzo y Cardone-Riportella, 2016). El supuesto detrás de estas intervenciones es que la mejora en el acceso al crédito de estas empresas permite generar inversiones que se traducen en el desarrollo y crecimiento de estas empresas. Además, dada la importancia que tienen las mipymes en la generación del producto interno bruto, impactan positivamente sobre el crecimiento de la economía y del empleo (Kersten et al., 2017; Butler et al., 2017). Este mecanismo está demostrado por el efecto dinamizador que tienen las mipymes en las economías en vías de desarrollo (Beck y Demirguc-Kunt, 2006; Berger y Udell, 2006; Beck et al., 2011; de la Torre et al., 2010).

Por otro lado, varios autores destacan que en Latinoamérica el acceso al financiamiento de las mipymes es bajo en relación con sus necesidades actuales y potenciales, por lo que existe una elevada demanda insatisfecha en la región (Llisterri et al., 2002). Las altas tasas de interés hacen no rentables sus proyectos de inversión y acotan su competitividad. Además, la escasa oferta de fondos provoca que las mipymes dependan del acceso al crédito de proveedores y de la disponibilidad de fondos propios para llevar adelante inversiones.

Los programas de apoyo financiero a las mipymes en Argentina han sido estudiados en mayor medida contando con datos provistos por los oferentes de distintos instrumentos aislados, así como el impacto a nivel empresa parcial. Sin embargo, la falta de datos de panel nacionales a nivel empresa y la imposibilidad de realizar estudios con grupos de control dificulta la evaluación profunda del impacto de las políticas (Kulfas, 2009; Castillo et al., 2016; Butler et al., 2017), a pesar de que 
Argentina cuenta con numerosos programas dotados de cierta continuidad desde los noventa (Lavarello y Sarabia, 2015).

El objetivo de este trabajo es analizar el alcance de las políticas públicas de apoyo financiero desde la perspectiva de la demanda de créditos a tasa subsidiada en Argentina. Para ello, se propone un enfoque novedoso, ya que no solo se pretende analizar las características de las empresas que solicitaron un crédito bancario a tasa subsidiada, sino que además se intenta evaluar la caracterización de las empresas que estarían dispuestas a solicitar créditos subsidiados para financiar una inversión, es decir, la demanda potencial.

Los aportes de este trabajo son diversos. En primer lugar, la profundización del estudio de la demanda efectiva revelaría si las políticas públicas alcanzan a las empresas con restricciones en el acceso al crédito bancario tradicional, es decir, necesidades insatisfechas de financiamiento en el pasado. En segundo lugar, estudiar las empresas demandantes potenciales permite analizar instrumentos de política que contengan herramientas que intensifiquen la inclusión de mipymes restringidas en el acceso al crédito, ampliando así la base de sujetos de créditos en el sistema bancario. Asimismo, los resultados de este trabajo sirven de guía para el diseño de políticas públicas, ya que provee un espacio para la discusión acerca de los destinatarios potenciales que no encuentran en el mercado crediticio instrumentos que les permitan cubrir sus necesidades financieras.

Este trabajo se estructura de la siguiente manera. Además de esta introducción, en la sección 2 se desarrolla la revisión de la literatura sobre la estructura de financiamiento de las mipymes, las políticas públicas que atienden sus problemas de financiamiento, y se construyen las hipótesis para abordar el trabajo empírico. En la sección 3 se aborda el contexto de las mipymes en Argentina y su financiamiento. En la sección 4 se presenta la metodología usada para el abordaje de la investigación, las características de la muestray las variables utilizadas. Por último, en la sección 5 se muestran los resultados del análisis empírico, y en la sección 6, las conclusiones del estudio.

\section{REVISIÓN DE LA LITERATURA}

\section{Los problemas de información y la estructura de financiamiento de las mipymes}

Las dificultades de acceso al financiamiento son explicadas por los problemas de información asimétrica que tienen lugar en los mercados de crédito 
(Jensen y Mecking, 1976). Uno de los principales trabajos en la literatura es el de Stiglitz y Weiss (1981), autores que encuentran que la información asimétrica genera dos fenómenos en los mercados financieros: los problemas de selección adversa y riesgo moral.

La selección adversa surge debido a que de los prestamistas no pueden distinguir entre buenos y malos prestatarios, en tanto desconocen los riesgos y la rentabilidad asociada a su proyecto (Akerlof, 1970). La tasa de interés en el mercado se corresponde con la de los proyectos de riesgo medio, lo cual provoca que los mejores proyectos se vean penalizados por una tasa de interés elevada e implica un costo superior a la rentabilidad esperada. Esto deriva en que se financien proyectos con mayor riesgo y rentabilidad asociados.

Por su parte, el riesgo moral sucede cuando, debido a las asimetrías de información, los contratos no se realizan. La dificultad de controlar las acciones de los deudores provoca altos costos para los prestamistas debido a la dificultad de monitoreo una vez concedido el préstamo, o debido al ocultamiento de información por parte de los prestatarios durante la solicitud del crédito.

Ante los problemas de información asimétrica, la forma de señalizar buenos proyectos es mediante la utilización de garantías. Por ello, las empresas con menor incertidumbre respecto al éxito de su proyecto están dispuestas a ofrecer garantías y demostrar que podrán afrontar sus obligaciones futuras sin ejecutarla. La solución a la ineficiencia de los mercados genera una barrera de entrada al sistema financiero, sobre todo para las empresas con escasa disponibilidad de activos físicos, jóvenes y pequeñas.

La estructura de financiamiento diferencial de las mipymes es explicada por distintas teorías, validadas en la literatura a nivel internacional. Entre las principales se encuentran las teorías del Pecking Order, del Trade-Off y del ciclo de crecimiento financiero. El enfoque del Pecking Order fue impulsado por los trabajos de Myers (1984) y Myers y Majluf (1984), y comprueba que existe un orden de preferencia en el uso de fuentes de financiamiento en función de su costo, su flexibilidad y autonomía. En el caso de los empresarios mipymes, ante la necesidad de financiar una inversión, esta teoría afirma que, en primer lugar, utilizarían fondos propios, luego fuentes externas de bajo costo y seguras (proveedores, clientes y créditos de familiares y amigos) y, por último, fuentes más costosas y/o instrumentos más complejos como bancos, mercados de capitales, bonos, etc. (Zoppa y McMahon, 2002). 
Por su parte, el enfoque del Trade-off agrupa distintos aportes que sostienen que existe un ratio de deuda-capital óptimo, el cual maximiza el valor de la empresa, por cuanto se equilibran los beneficios y costos de tomar deuda. Estas teorías consideran los efectos de los impuestos corporativos y personales, de los costos directos e indirectos de quiebra y de los costos que surgen por los problemas de agencia (Modigliani y Miller, 1958; Modigliani y Miller, 1963; Miller, 1977; entre otros). De acuerdo con estos aportes, se espera una relación positiva entre el endeudamiento y el valor actual del escudo fiscal, y una relación negativa entre el ratio de deuda y los costos de quiebra y los costos originados por los problemas de agencia. Por lo tanto, dependiendo de las condiciones de la empresa, el endeudamiento puede reportar beneficios.

El último aporte ampliamente testeado empíricamente para explicar la estructura de financiamiento de las mipymes es el enfoque planteado por Berger y Udell (1998), conocido como el ciclo de crecimiento financiero. Según estos autores, las mipymes se caracterizan por financiarse con distintas fuentes dependiendo de su tamaño, su antigüedad y la información fidedigna que pueden generar y ofrecer sobre su actividad comercial y financiera. Describen así que las microempresas no cuentan con el aval de garantías para sus operaciones financieras ni historial crediticio, se financian con fondos propios, especialmente con fondos pertenecientes a los propietarios y fuentes informales (familiares y amigos, préstamos a sola firma, inversores ángeles, entre otros). Las pequeñas empresas, con alto crecimiento potencial y sin historial crediticio pueden acceder además a financiarse con créditos comerciales (proveedores o clientes), y en economías con mercados financieros desarrollados, con fondos de capital de riesgo. Entre tanto, las medianas empresas con historial crediticio, debido a la sistematización de varios años de operaciones comerciales y financieras, y con activos para ofrecer como garantías, acceden a un abanico de fuentes más diverso y formal. Entre estas se encuentran los préstamos otorgados por instituciones financieras a corto, mediano y largo plazo.

La evidencia empírica de estas teorías sustenta la relación positiva entre el tamaño de la empresa y el acceso al financiamiento. En este sentido, las empresas pequeñas reportan en mayor medida problemas para financiarse como el principal obstáculo de su operatoria (Liu y Tian, 2009; Forte et al., 2013; Dong y Men, 2014). Wang (2016) argumenta que el acceso al financiamiento es el mayor obstáculo que presentan las mipymes de los países en vías de desarrollo, siendo la antigüedad y el tamaño de la empresa características determinantes para abordar esta conclusión. Similar evidencia ya ha sido demostrada por otros autores (Berger y Udell, 1998; Beck y Demirgüc-Kunt, 2006; Bebczuk, 2010; Dong y Men, 2014). 
Bebczuk (2004), a partir de una regresión logística, estudia los determinantes del acceso al crédito de las mipymes argentinas, con base en una encuesta de 665 respuestas, realizada en 1999 por la Unión Industrial. Las variables explicativas utilizadas fueron: edad de la empresa, nivel de ventas (como proxy de tamaño), años de relación con el banco principal, si tiene relación con un banco cooperativo, uso de sobregiro, expectativas de crecimiento de las exportaciones, expectativas de crecimiento de la producción, plazo de crédito comercial otorgado a clientes; y variables de la hoja de balance como: retorno sobre activos (ROA), disponibilidad de activos fijos (activos fijos/activos totales, proxy colateral), liquidez (efectivo/activos totales), deuda/activos. Entre los resultados encontrados, el tamaño no es significativo para determinar la probabilidad de obtener un préstamo, la rentabilidad de la empresa la afecta en forma positiva, la liquidez en forma negativa, mayores ratios de deuda aumentan la probabilidad de conseguir un préstamo, y el uso de sobregiros también la afectan en forma positiva.

Respecto a la relación entre la antigüedad de la empresa y el acceso al financiamiento, la evidencia no es concluyente. Algunos trabajos muestran que las restricciones financieras están inversamente asociadas con la antigüedad (Petersen y Rajan, 1994; Kumar y Francisco, 2005); otros autores afirman que existe una relación positiva (Gregory et al., 2005; Bougheas et al., 2006); otros más no encuentran ninguna relación entre estas dos variables (Cole y Dietrich, 2012; Guercio et al., 2014).

En las empresas familiares y de capital cerrado, las preferencias y los objetivos de sus dueños adquieren relevancia en la determinación de su estructura de capital (Romano et al., 2001). Por lo tanto, la edad del propietario-gerente, su género y su nivel de educación pueden ser características relevantes para explicar las decisiones de financiamiento. Las empresas familiares se muestran más adversas a la toma de fuentes de financiamiento externo más complejas que el crédito ofrecido por la banca comercial, como pueden ser inversores ángeles o mercado de capitales, porque temen a la pérdida de control de su empresa (Storey, 1994). La edad del propietario-gerente está asociada negativamente con el uso de deuda de corto plazo (Hutchison, 2004). Por su parte, las empresas conducidas por mujeres, en general, son más reticentes al uso de fuentes externas de financiamiento (Carter y Rosa, 1998) y presentan mayores dificultades en el acceso (Guercio et al., 2015). Los propietarios que tienen por objetivo el desarrollo de su empresa y el crecimiento de sus ganancias conciben el endeudamiento como positivo, mientras que aquellos que no persiguen esos objetivos prefieren el autofinanciamiento (Romano et al., 2001; Briozzo y Vigier, 2009a). 
Otros autores encontraron que la falta de acceso a fuentes externas de financiamiento está vinculada al fenómeno de autoexclusión (Briozzo y Vigier, 2006; Bebczuc, 2010; Freel et al., 2012; Cole, 2016). Según estos autores, las pymes no demandan crédito no porque no tengan proyectos de inversión rentables, sino por razones como requisitos de documentación engorrosos, plazos y tasas no acordes, desconocimiento de las fuentes, o porque consideran que no serán declarados sujetos de crédito por la entidad bancaria.

El marco teórico desarrollado se completa con aportes de otros autores que encuentran limitantes endógenas para acceder al financiamiento externo de las mipymes y que provocan la intervención del Gobierno. Los aportes tienen su origen en el trabajo de Steindl (1945), donde desarrolla el principio de riesgo creciente, según el cual las empresas de menor tamaño no tienen la misma capacidad de acumulación de capital que las empresas de gran tamaño, por lo que su estructura de activos no les permite hacer frente a inversiones y deben recurrir al uso de fondos externos. Esto sucede en un contexto de mercado oligopólico en la producción, donde coexisten empresas grandes y pequeñas. Las grandes mantienen escalas de producción e inversión cuasi monopólicas que las pequeñas no disponen, sumado a sus restricciones financieras, lo que ocasiona que las pequeñas no tengan posibilidad de crecimiento.

\section{Los programas de políticas públicas para suavizar las restricciones financieras}

La intervención del Estado en los mercados financieros procura su mejor funcionamiento, lo que se traduce en mejoras en los resultados para el conjunto de la economía (Stiglitz, 1993). Dada la existencia de problemas de acceso al financiamiento de las mipymes, los gobiernos instrumentan distintos programas de políticas públicas tendientes a solucionar las fallas de los mercados financieros, exacerbadas para este conjunto de empresas. En esta sección se presenta la evidencia recogida por la literatura sobre el impacto y el alcance de la intervención del Estado en los mercados financieros, así como estudios que analizan la demanda de créditos bancarios y promocionales, es decir, aquellos vinculados a programas públicos de financiamiento.

\section{El impacto y alcance de los programas de políticas públicas de financiamiento en las mipymes}

Dentro de las medidas que evalúan el desempeño de las políticas públicas, se encuentran aquellas que miden el impacto de estas. La literatura ha crecido en los últimos 
años y entre los trabajos se destacan los aportes de Grimm y Pauffhausen (2015), Cravo y Piza (2016), Kersten et al. (2017), Butler et al. (2017) y Bueso-Merriam et al. (2016). El objetivo, la metodología empleada, así como los principales resultados del estudio de estos trabajos se sintetizan en la tabla 1.

Tabla 1.

Estudios sobre el impacto de programas de política pública de financiamiento en las mipymes

\begin{tabular}{|c|c|c|}
\hline Autores (año) & Metodología y objetivo & Resultados \\
\hline $\begin{array}{c}\text { Grimm y } \\
\text { Pauffhausen } \\
\text { (2015) }\end{array}$ & $\begin{array}{l}\text { Revisión sistemática de la } \\
\text { literatura focalizada en el } \\
\text { impacto de los programas } \\
\text { dirigidos a mipymes sobre la } \\
\text { creación de empleo en países en } \\
\text { vías de desarrollo. }\end{array}$ & $\begin{array}{l}\text { Encuentran que las intervenciones en el acceso } \\
\text { al financiamiento generan un bajo efecto sobre } \\
\text { el empleo y que la repercusión de estas políticas } \\
\text { es mayor en empresas nuevas que en empresas } \\
\text { más antiguas. }\end{array}$ \\
\hline $\begin{array}{c}\text { Cravo y Piza } \\
\text { (2016) }\end{array}$ & $\begin{array}{l}\text { Meta-análisis para sintetizar } \\
\text { los efectos encontrados por la } \\
\text { evidencia empírica respecto a } \\
\text { los resultados de la evaluación } \\
\text { de políticas públicas dirigidas a } \\
\text { mipymes. }\end{array}$ & $\begin{array}{l}\text { Este estudio señala que los distintos tipos de } \\
\text { programas que asisten a las mipymes mejoran } \\
\text { su performance y provocan un impulso sobre } \\
\text { la creación de empleo y la productividad de la } \\
\text { mano de obra. }\end{array}$ \\
\hline $\begin{array}{l}\text { Kersten et al. } \\
\quad \text { (2017) }\end{array}$ & $\begin{array}{l}\text { Revisión sistemática de la } \\
\text { literatura y meta-análisis para } \\
\text { conocer acerca de la efectividad } \\
\text { de los programas que proveen } \\
\text { acceso al financiamiento a } \\
\text { mipymes. }\end{array}$ & $\begin{array}{l}\text { Los programas de financiamiento para mipymes } \\
\text { tienen un efecto positivo y significativo sobre } \\
\text { los montos financiados y sobre la inversión. } \\
\text { Además, observan que la mayoría de los } \\
\text { trabajos encuentran efectos positivos de los } \\
\text { programas financieros sobre el desempeño de } \\
\text { la empresa en términos de producto, empleo, } \\
\text { ventas, beneficios y valor agregado. }\end{array}$ \\
\hline Butler et al. (2017) & $\begin{array}{l}\text { Analiza el impacto de tres } \\
\text { programas en Argentina: Fondo } \\
\text { Nacional de Desarrollo para } \\
\text { la Micro, Pequeña y Mediana } \\
\text { Empresa (Fonapyme), Régimen } \\
\text { de Bonificación de Tasas (RBT) } \\
\text { y Sociedades de Garantía } \\
\text { Recíproca (SGR). } \\
\text { Método de tratamiento de datos: } \\
\text { técnicas de emparejamiento } \\
\text { estadístico con efectos fijos, } \\
\text { estimación por mínimos } \\
\text { cuadrados ordinarios. }\end{array}$ & $\begin{array}{l}\text { Estos autores concluyen que las empresas } \\
\text { beneficiarias de estos programas se caracterizan } \\
\text { por tener un mayor tamaño (medido por } \\
\text { cantidad de empleados), y haber accedido a } \\
\text { créditos en períodos anteriores. } \\
\text { Los sectores comercio e industria son los } \\
\text { sectores que más empresas beneficiarias } \\
\text { concentran. El impacto sobre la productividad } \\
\text { de los programas es mayor en las empresas } \\
\text { jóvenes que en las empresas más antiguas, } \\
\text { lo cual sugiere que es correcto el canal de } \\
\text { transmisión de política pública (empresas } \\
\text { jóvenes con menor acceso al crédito y con } \\
\text { potencial de crecimiento). }\end{array}$ \\
\hline
\end{tabular}




\begin{tabular}{|c|c|c|}
\hline Autores (año) & Metodología y objetivo & Resultados \\
\hline $\begin{array}{c}\text { Bueso-Merriam } \\
\text { et al. (2016) }\end{array}$ & $\begin{array}{l}\text { Analizan el impacto del } \\
\text { Programa de Crédito para el } \\
\text { Desarrollo de la Producción y el } \\
\text { Empleo, en la Provincia de San } \\
\text { Juan, Argentina, entre los años } \\
2004 \text { y } 2014 \text {. } \\
\text { Método de tratamiento de } \\
\text { datos: modelo dinámico de } \\
\text { variable dependiente rezagada, } \\
\text { estimación por mínimos } \\
\text { cuadrados ordinarios. }\end{array}$ & $\begin{array}{l}\text { Los autores encontraron efectos positivos } \\
\text { sobre las ventas, la generación de empleo y la } \\
\text { productividad de las empresas beneficiarias. } \\
\text { Cuando se controla por tamaño, se encontraron } \\
\text { efectos positivos para las empresas pequeñas } \\
\text { (hasta } 20 \text { empleados) en la inversión, ventas } \\
\text { y productividad, y en las empresas medianas } \\
\text { (más de } 21 \text { empleados), se encontró un efecto } \\
\text { positivo en ventas y empleo muy superior } \\
\text { al grupo anterior. Respecto a las empresas } \\
\text { clasificadas por sector de actividad, se } \\
\text { hallaron efectos positivos y significativos en } \\
\text { las variables inversión, ventas y empleo en el } \\
\text { sector agricultura, muy superiores a los de los } \\
\text { otros dos sectores considerados (industria } \\
\text { y comercio). En el sector industria solo se } \\
\text { encontró significativa la variable inversión, } \\
\text { y en el sector comercio, ninguna variable } \\
\text { considerada fue significativa. }\end{array}$ \\
\hline
\end{tabular}

Fuente: elaboración propia.

Particularmente, en Argentina se encuentran estudios aislados sobre los programas de apoyo financiero, con datos provistos por agencias públicas oferentes de instrumentos; es también limitado el estudio del impacto a nivel empresa debido a la falta de datos de panel nacionales. Por estas razones, se reconoce la dificultad de realizar estudios con grupos de control y evaluar el impacto de las políticas en esta temática (Kulfas, 2009; Castillo et al., 2016; Butler et al., 2017). En la tabla 1 destacamos dos trabajos actuales: Butler et al. (2017) y Bueso-Merriam et al. (2016), que estudian el impacto de programas de apoyo al financiamiento en mipymes argentinas.

Por otro lado, si bien varios trabajos mencionan que en Argentina el alcance de las políticas públicas de apoyo a las mipymes es bajo, no se especifica qué se entiende por "alcance". Este término permite abordar el estudio de los programas de políticas públicas desde otros aspectos, no solo considerando la eficiencia propia del programa respecto a otros programas o a la eficacia en el universo afectado, sino considerando el entorno socioeconómico donde se desarrollan las mipymes.

Ferraro y Stumpo (2010) definen alcance como la relación entre el universo de potenciales beneficiarios y la población total de mipymes; destacan que, en promedio, en América Latina, este porcentaje no llega al 15\%. Un documento del Ministerio de Economía y Finanzas Públicas (2011) indica que el alcance de los instrumentos de política pública es "la correspondencia que desde su diseño podría presentar con sectores de actividad ('sectorial') específicos" (p. 16). A este grupo de instrumentos 
se le denomina de "alcance sectorial", son las "herramientas que promueven un sector económico en particular, sea que este se presente en forma agregada (agro, industria, comercio o servicios) o específica (sector autopartista, editorial, etc.)" (p. 32). Por otro lado, el alcance horizontal se "refiere a aquellos instrumentos que no limitan la promoción a sectores de actividad concretos, ya que abarcan horizontalmente la actividad productiva" (p. 32). Por último, en este informe se menciona una dimensión adicional: la geográfica, que se concibe como "la posibilidad de circunscribir su rango de acción a una extensión territorial específica” (p. 33).

Goldstein (2011) menciona el bajo alcance de algunos programas en términos territoriales. La autora define alcance como la llegada del programa objeto de análisis (Programa para el Desarrollo Regional y Sectorial [PRODER], línea de créditos otorgados por el Banco de la Nación Argentina) a todas las provincias y a la mayor cantidad de beneficiarios. Esta llegada depende de la difusión y selección de beneficiarios por parte de las organizaciones intermediarias y los técnicos asesores. El seguimiento de los proyectos aprobados para dimensionar su impacto regional consistió en su evaluación respecto a la creación de empleo, o sobre mejoras medioambientales, o en la calidad de vida de los habitantes, además de evaluar el impacto a nivel de empresa respecto al nivel de facturación y número de empleados.

En este trabajo se sigue el concepto de alcance de Ferraro y Stumpo (2010), este se define como una relación entre el número de beneficiarios y el universo de mipymes, en un territorio determinado. A este concepto se asignan diversos niveles, considerando el mayor grado de alcance cuando la empresa demanda efectivamente un programa público de financiamiento y un nivel de alcance anterior cuando la empresa se presenta como potencialmente demandante.

\section{Estudios sobre la demanda de créditos bancarios y promocionales}

En general, diversos estudios indican que los problemas de acceso al crédito mipymes radican en las restricciones impuestas por los oferentes de crédito en cuanto a plazos, tasas, exigencias de garantías y documentación (Cowling et al., 2016). Otros autores, en cambio, encuentran que es menos frecuente ser rechazado en una solicitud de crédito bancario que no aplicar a un crédito bancario por miedo a ser rechazado (Kon y Storey, 2003; Freel et al., 2012; Cole y Sokolyk, 2016).

En este sentido, Bebczuk (2010) asegura, con base en datos de pymes argentinas, que una proporción considerable de pymes no demandan créditos y que "el Estado no ha logrado en absoluto reparar los problemas del crédito para las pymes" (Bebczuk, 
2010, p. 8), en tanto las pymes tienen un bajo conocimiento de los programas públicos y demuestran una baja satisfacción respecto a los procedimientos de acceso y adjudicación de los beneficios. Este autor sostiene que la asistencia financiera estatal debería estar dirigida a las empresas que presentan restricción crediticia; la define como la situación en la que una empresa "ve rechazada su solicitud de préstamo a pesar de satisfacer las siguientes tres condiciones: i) cuenta con proyectos de alta rentabilidad esperada y por tanto con suficiente capacidad de pago; ii) no posee suficientes fondos propios para encarar tales proyectos; y iii) tiene los incentivos correctos y la voluntad de honrar el compromiso financiero asumido" (Bebczuk, 2010, p. 12).

Con el objetivo de identificar los trabajos que analizan los problemas de acceso al financiamiento del lado de la demanda, y luego centrar la discusión en el objeto de estudio de este trabajo: los créditos a tasa subsidiada, se expone una breve síntesis de la literatura en esta sección. La tabla 2 muestra un resumen de una selección de trabajos existentes en la literatura acerca de la demanda potencial de créditos bancarios.

En relación con la demanda de créditos promocionales, uno de los escasos trabajos en la literatura que plantea una discusión sobre esta temática es el desarrollado por Briozzo y Vigier (2009b), autores que estudian la demanda de créditos promocionales para una muestra de pymes argentinas. Los autores observan que entre las principales limitaciones de los instrumentos de apoyo financiero se destaca el hecho de canalizarse a través del sistema bancario, lo que implica que las pymes atraviesan las mismas barreras de entrada que en la solicitud de un crédito bancario tradicional. Estas barreras se asocian principalmente a las características de informalidad de las pymes (falta de registros formales, situación fiscal y previsional irregular) y la dificultad técnica de las solicitudes (desconocimiento de la confección de planes de negocio con sus proyecciones económicas y financieras). Los autores encuentran que tamaño y reinversión de utilidades tienen un efecto positivo sobre la demanda de créditos promocionales, mientras que el nivel de educación de los propietarios tiene un efecto negativo.

El presente trabajo pretende ampliar la investigación sobre la demanda de créditos promocionales a tasa subsidiada e indagar acerca de la demanda potencial. A los propósitos de esta investigación, demanda potencial se define como el universo de mipymes que aplicarían a una convocatoria de créditos a tasa subsidiada para financiar una inversión. Esta definición se corresponde con la actitud de estas empresas frente a este tipo de fuentes de financiamiento, mas no con su accionar real. 
Tabla 2.

\section{Estudios sobre la demanda potencial de crédito bancario}

\begin{tabular}{|c|c|c|}
\hline Autor/es (año) & Definición de demanda potencial & Método, objetivo y resultados \\
\hline $\begin{array}{l}\text { Rostamkalaei } \\
\text { (2017) }\end{array}$ & $\begin{array}{l}\text { Define la demanda potencial de crédito } \\
\text { como el conjunto de aquellos agentes } \\
\text { que no aplican a un crédito, pero } \\
\text { desean hacerlo y no pueden por temor } \\
\text { a ser rechazados en sus solicitudes. }\end{array}$ & $\begin{array}{l}\text { Con base en la encuesta UK SME Finance Monitor } \\
\text { (2011-2016) y modelos de regresión Probit, evalúan } \\
\text { el efecto del tiempo transcurrido desde la crisis } \\
\text { financiera sobre la probabilidad de no demandar } \\
\text { crédito. Encuentra una relación significativa y } \\
\text { positiva con la edad del propietario cuando es mayor } \\
\text { a } 50 \text { años y respecto al sector de actividad, y negativa } \\
\text { con el tamaño de la empresa y su antigüedad. }\end{array}$ \\
\hline $\begin{array}{l}\text { Freel et al. } \\
\quad \text { (2012) }\end{array}$ & $\begin{array}{l}\text { Consideran que existe una demanda } \\
\text { potencial en los prestatarios que no } \\
\text { aplican a un crédito bancario por } \\
\text { temor a ser rechazados (los denominan } \\
\text { discouraged borrowers). }\end{array}$ & $\begin{array}{l}\text { Los resultados de su análisis multivariado indican } \\
\text { que existen diferencias entre el grupo de empresas } \\
\text { que son rechazadas de las que desisten de aplicar. } \\
\text { La probabilidad de pertenecer al grupo de las } \\
\text { desalentadas disminuye conforme aumenta el } \\
\text { tamaño de la empresa, si se trata de una empresa } \\
\text { familiar, si la empresa pertenece a una industria de } \\
\text { producción o comercial, y tener una relación activa } \\
\text { con el sector bancario. Mientras ser un emprendedor } \\
\text { recurrente y tener una forma legal que limita } \\
\text { responsabilidad aumentan la probabilidad de desistir } \\
\text { de aplicar a un crédito bancario. }\end{array}$ \\
\hline $\begin{array}{l}\text { Cole y Sokolyk } \\
\text { (2016) }\end{array}$ & $\begin{array}{l}\text { La demanda potencial se identifica } \\
\text { en este trabajo con la categoría de } \\
\text { discouraged firms: grupo de empresas } \\
\text { que necesitan crédito bancario y } \\
\text { no demandan porque esperan ser } \\
\text { rechazadas. }\end{array}$ & $\begin{array}{l}\text { Estudian las características de la demanda de } \\
\text { crédito con base en datos de la encuesta Small } \\
\text { Business Finances, realizada en los años 1993, } 1998 \\
\text { y } 2003 \text { en Estados Unidos. El grupo de empresas } \\
\text { que necesita crédito bancario y no demandan son } \\
\text { más pequeñas, tienen una calificación crediticia } \\
\text { inferior, sus propietarios son de mayor edad, y usan } \\
\text { pocos servicios financieros, respecto a quienes } \\
\text { aplican. Además, encuentran que las empresas que } \\
\text { no solicitaron difieren del grupo de las rechazadas } \\
\text { en varias características: son más pequeñas, más } \\
\text { antiguas, más rentables, sus dueños son de mayor } \\
\text { edad y utilizan pocos servicios financieros. }\end{array}$ \\
\hline
\end{tabular}

Fuente: elaboración propia.

\section{LAS MIPYMES EN ARGENTINA Y SU FINANCIAMIENTO}

Dentro de las denominadas micro, pequeñas y medianas empresas (mipymes), se encuentra alrededor del 99\% del universo empresarial de la República Argentina. Si las diferenciamos por tamaño, el $71 \%$ de las empresas registradas son clasificadas como microempresas, el $24 \%$ como pequeñas y el $6 \%$ como medianas. ${ }^{1}$ Este conjunto

1 La clasificación por tamaño de empresa en Argentina tiene en cuenta variables como el nivel de ventas, el número de empleados y el volumen de activos, clasificados por la actividad económica. 
heterogéneo explica la producción del 64\% del empleo formal del país, según datos del Ministerio de Producción y Trabajo de la Nación. Sin embargo, son reconocidas las dificultades en el acceso al financiamiento externo que tienen estas empresas en comparación con las grandes. Argentina se caracteriza por tener un mercado financiero con escasa profundidad. El crédito interno destinado al sector privado no financiero es de un 16\% como porcentaje del Producto Bruto Interno (PBI), siendo del 25\% para Latinoamérica, según datos del Banco Mundial para el 2017. Este porcentaje se reduce al 3\% del PBI si se estima para las mipymes (Unión Industrial Argentina [UIA], 2018).

Según estimaciones del Ministerio de Hacienda de la Nación (2019), el 37,4\% de las empresas accede a financiamiento, y si se incluyen los trabajadores por cuenta propia, esta proporción se reduce a $14,5 \%$ del total de mipymes. La principal fuente externa utilizada es el financiamiento bancario, al cual recurren el 38\% de las mipymes y representa el $94 \%$ de sus recursos externos. Respecto al destino de estos fondos, para el 2018, el 87\% del crédito se empleó para capital de trabajo y un $13 \%$ para la financiación de inversión.

Desde mediados de los noventa, con la sanción de la Ley PyME (24.467), en Argentina se instrumentaron diversos programas de apoyo al financiamiento a las mipymes. Entre los más difundidos se encuentran los créditos a tasa subsidiada como el Régimen de Bonificación de Tasas y la Línea de Financiamiento para la Producción y la Inclusión Financiera; ambos programas representaban el 52\% de los créditos a pymes en el 2017, equivalentes a 8000 millones de dólares (UIA, 2018). Sin embargo, pese a la continuidad de los programas para el financiamiento de mipymes, su alcance es limitado, dada su baja utilización y acceso (Bebczuk, 2010). Además, la falta de un sistema de monitoreo y control que permita contar con datos para el análisis imposibilita la evaluación de las políticas públicas mencionadas, así como la medición de su impacto (Kulfas, 2009).

\section{DATOS Y METODOLOGÍA}

Los datos utilizados en este trabajo surgen de una base propia que recolecta información acerca de los problemas de acceso al financiamiento en la región del sudoeste de la Provincia de Buenos Aires en la República Argentina, entre finales del 2017 y principios del 2018. ${ }^{2}$ La muestra contiene un total de 285 mipymes. La descripción

2 Para determinar el tamaño de la muestra, se utilizó un muestreo aleatorio simple a partir de la población identificada en el listado público de empresas activas. 
de las variables empleadas en este estudio se consolida en la tabla 3. En primer lugar, se presentan las variables dependientes del estudio, demanda efectiva (DE) y demanda potencial (DP) de créditos a tasa subsidiada (CTS). En segundo lugar, se presentan las variables vinculadas al perfil de la empresa y del empresario. Por último, se muestran las variables de la estructura financiera y aquellas que identifican los problemas de acceso al financiamiento de las mipymes.

Tabla 3.

Descripción de las variables

\begin{tabular}{|c|c|}
\hline Variable & Descripción \\
\hline \multicolumn{2}{|r|}{ Variables dependientes } \\
\hline $\mathrm{DE}$ & $\begin{array}{l}\text { Variable binaria: toma valor } 1 \text { si la empresa ha solicitado alguna línea CTS en el } \\
\text { último tiempo, } 0 \text { en caso contrario. }\end{array}$ \\
\hline DP & $\begin{array}{l}\text { Variable binaria: toma valor } 1 \text { si la empresa elegiría como opción financiar una } \\
\text { nueva inversión en un activo fijo mediante un CTS y no tomó un CTS en los últimos } \\
\text { años }(\mathrm{DE}=0), 0 \text { en caso contrario. }\end{array}$ \\
\hline \multicolumn{2}{|r|}{ Variables explicativas } \\
\hline \multicolumn{2}{|r|}{ Características de la empresa } \\
\hline Tamaño & $\begin{array}{l}\text { Variable ordinal: toma valor } 1 \text { si la empresa es micro, } 2 \text { si es pequeña y } 3 \text { si la } \\
\text { empresa es mediana. }\end{array}$ \\
\hline Antigüedad & Variable continua: se corresponde con la edad de la empresa medida en años. \\
\hline Sector & $\begin{array}{l}\text { Variable nominal: toma valor } 1 \text { si la empresa pertenece al sector de actividad } \\
\text { servicios, } 2 \text { al comercial, } 3 \text { al industrial, } 4 \text { al sector construcción. }\end{array}$ \\
\hline Forma jurídica & $\begin{array}{l}\text { Variable nominal: toma valor } 1 \text { si la empresa pertenece al sector de actividad } \\
\text { servicios, } 2 \text { al comercial, } 3 \text { al industrial, } 4 \text { al sector construcción. }\end{array}$ \\
\hline Empresa familiar & $\begin{array}{l}\text { Variable binaria: toma valor } 1 \text { si el } 50 \% \text { o más de la empresa pertenece a una } \\
\text { familia, o si uno o varios miembros controlan efectivamente el negocio, y/o si la } \\
\text { segunda generación trabaja en la empresa. }\end{array}$ \\
\hline \multicolumn{2}{|r|}{ Características de los propietarios-gerente } \\
\hline Edad & Variable continua: indica la edad del gerente-propietario. \\
\hline Educación & $\begin{array}{l}\text { Variable ordinal: indica el nivel máximo de educación alcanzado por el gerente- } \\
\text { propietario. Toma valor } 1 \text { si tiene nivel de educación primario, } 2 \text { si tiene nivel medio } \\
\text { y } 3 \text { si tiene nivel de educación superior (terciario, universitario o posgrado). }\end{array}$ \\
\hline Objetivo empresario & $\begin{array}{l}\text { Variable binaria: toma valor } 1 \text { si el objetivo principal del propietario respecto } \\
\text { a su empresa es empresarial: aumentar el valor del negocio, o lograr mayor } \\
\text { participación en el mercado, y } 0 \text { si el objetivo más importante es de carácter } \\
\text { personal: dar trabajo a los miembros de su familia, generar ingresos para su familia } \\
\text { o pasar el negocio a la siguiente generación. }\end{array}$ \\
\hline
\end{tabular}

3 Se asigna el tamaño otorgado en el "Certificado PyME" en el caso de que la empresa haya accedido al "nuevo registro PyME” (Formulario F 1272 de la Administración Federal de Ingresos Públicos [AFIP]). En caso de no estar registrada, se calcula su tamaño a partir del volumen de ventas y el sector de pertenencia según la Resolución del Ministerio de Producción n. ${ }^{\circ}$ 103/17, medida en pesos argentinos. 


\begin{tabular}{|c|c|}
\hline Variable & Descripción \\
\hline \multicolumn{2}{|r|}{ Estructura de financiamiento } \\
\hline Fondos propios & $\begin{array}{l}\text { Variable binaria: toma valor } 1 \text { si la empresa usa habitualmente como fuente de } \\
\text { financiamiento utilidades reinvertidas o aportes de los socios. }\end{array}$ \\
\hline Crédito comercial & Variable binaria: toma valor 1 si la empresa usa crédito de sus proveedores. \\
\hline Crédito bancario & $\begin{array}{l}\text { Variable binaria: toma valor } 1 \text { si la empresa usa crédito bancario de corto y/o largo } \\
\text { plazo. }\end{array}$ \\
\hline $\begin{array}{l}\text { Otro financiamiento no } \\
\text { bancario }\end{array}$ & $\begin{array}{l}\text { Variable binaria: toma valor } 1 \text { si la empresa usa créditos de prestamistas privados, } \\
\text { de otras empresas privadas, créditos otorgados por instituciones públicas o } \\
\text { privadas no bancarias, o préstamos de familiares, amigos o conocidos. }\end{array}$ \\
\hline \multicolumn{2}{|r|}{ Variables indicativas de problemas de acceso al financiamiento } \\
\hline $\begin{array}{l}\text { Exclusión al crédito } \\
\text { bancario }\end{array}$ & $\begin{array}{l}\text { Variable binaria: toma valor } 1 \text { si la empresa ha mostrado, en los últimos } 3 \text { años, } \\
\text { necesidades de crédito insatisfechas. Dentro de este grupo de empresas se } \\
\text { encuentran aquellas que solicitaron créditos bancarios y no accedieron, y aquellas } \\
\text { empresas que solicitarían créditos bancarios si cambian las características y las } \\
\text { condiciones de acceso como tasas, garantías, plazos, trámites, etc. }\end{array}$ \\
\hline Pérdida de inversión & $\begin{array}{l}\text { Variable binaria: toma valor } 1 \text { si la empresa se ha perdido de hacer inversiones o } \\
\text { encarar proyectos rentables por falta de fondos en los años } 2016 \text { y } 2017 .\end{array}$ \\
\hline
\end{tabular}

Fuente: elaboración propia.

El análisis empírico se realiza, en primer lugar, a partir de un estudio bivariado de contraste de medias y proporciones entre las variables dependientes y las variables explicativas que caracterizan el perfil de la empresa y su estructura de financiamiento. Para el tratamiento de los datos se aplicarán los test de Pearson Chi2 y Fisher's Exact para analizar la independencia entre dos variables categóricas. Cuando la variable dependiente es cuantitativa, para analizar la relación entre las variables, se aplican métodos paramétricos, $t$ de student o ANOVA, y no paramétricos, U de Mann-Whitney y Prueba de Kruskal-Wallis, dependiendo del supuesto sobre la distribución de la variable cuantitativa.

En segundo lugar, se construye un modelo de regresión logística para identificar el impacto de las variables independientes sobre la probabilidad de que una empresa sea demandante efectiva o potencial de créditos a tasa subsidiada. Los coeficientes estimados por modelo Logit muestran solo la existencia de la relación entre las variables independientes y las explicativas, y el signo de esta. Por ello, en este trabajo se calculan los efectos marginales entre las variables para identificar el impacto de cada variable independiente sobre la dependiente (Agresti, 2002). 


\section{ANÁLISIS EMPÍRICO}

\section{Estadísticas descriptivas}

La tabla 4 muestra la descripción de las variables explicativas. En primer lugar, se observa que solo el $11,2 \%$ de las empresas de la muestra (32/285) demandaron efectivamente ( $\mathrm{DE}=1$ ) un CTS (crédito con tasa subsidiada). Del 88,9\% que no demandó efectivamente este tipo de instrumentos (DE $=0$ ), el 59\% de empresas afirman que, ante una oportunidad de inversión en activos fijos, la forma de financiar la inversión sería un CTS. A este conjunto de empresas se denomina demanda potencial (DP = 1). En segundo lugar, se presentan los resultados de las estadísticas descriptivas y prueba de hipótesis, con el objetivo de identificar las características de las empresas que demandaron efectivamente un CTS y las empresas que demandarían potencialmente este tipo de instrumento.

En relación con la demanda efectiva, se observa que en el grupo de las demandantes $(\mathrm{DE}=1$ ) hay una menor participación de empresas micro y una mayor participación de empresas medianas que en el grupo de las no demandantes $(\mathrm{DE}=0)$. Este resultado podría estar indicando que el tamaño de la empresa se encuentra positivamente correlacionado con la demanda efectiva de este tipo de créditos. Las empresas que demandaron CTS son más antiguas, y hay un mayor porcentaje de empresas con formas jurídicas que limitan la responsabilidad patrimonial respecto a las empresas que no demandaron este instrumento. En cuanto al sector de actividad, se observa que hay una mayor participación de empresas de los sectores comercio y servicio en la demanda efectiva, y no se encontraron diferencias significativas en el resto de los sectores. Tampoco se encuentran diferencias significativas en cuanto a la estructura familiar de la empresa, ni en las variables relacionadas con las características del propietario/gerente.

Respecto a las variables financieras, se observa una mayor participación de empresas con créditos bancarios dentro del grupo de demandantes efectivas ( $D E=1$ ), y la participación de las empresas que consideran estar excluidas del crédito bancario es menor dentro de este grupo en comparación con las no demandantes $(\mathrm{DE}=0)$. Además, a excepción de un caso, el resto de las empresas de demanda efectiva ya estaban bancarizadas antes de solicitar un crédito a tasa subsidiada, es decir, no tenían necesidades de financiación bancaria. La variable que indica la pérdida de inversión por no contar con recursos financieros presenta una relación negativa y significativa con la demanda efectiva. 
Lilia José Corzo • María Belén Guercio • Hernán Pedro Vigier

Tabla 4.

Descripción de la muestra total, demanda efectiva y potencial de CTS

\begin{tabular}{|c|c|c|c|c|c|c|c|c|}
\hline & & & Den & da efecti & (DE) & Dem & la poten & (DP) \\
\hline Variables & explicativas & total & $\mathrm{DE}=0$ & $\mathrm{DE}=1$ & $\begin{array}{c}\text { Pearson } \\
\text { chi test }\end{array}$ & $\mathrm{DP}=0$ & $\mathrm{DP}=1$ & $\begin{array}{c}\text { Pearson } \\
\text { chi test }\end{array}$ \\
\hline & N & 285 & 253 & 32 & & 105 & 148 & \\
\hline & Micro & $53 \%$ & $57,3 \%$ & $15,6 \%$ & $(+)^{* * *}$ & $65,7 \%$ & $51,3 \%$ & $(+)^{* *}$ \\
\hline Tamaño & Pequeña & $32 \%$ & $30,8 \%$ & $37,5 \%$ & - & $22 \%$ & $37,1 \%$ & - \\
\hline & Mediana & $16 \%$ & $11,9 \%$ & $46,9 \%$ & - & $12,4 \%$ & $11,5 \%$ & - \\
\hline Ant & üedad & 27 & 26 & 33 & $(+)^{*}$ & 24 & 27 & $(+)^{*}$ \\
\hline Form & jurídica & $38 \%$ & $34 \%$ & $68,7 \%$ & $(+)^{* * *}$ & $19 \%$ & $44,6 \%$ & $(+)^{* * *}$ \\
\hline & Comercio & $56 \%$ & $56,9 \%$ & $46,9 \%$ & NS & $54,3 \%$ & $58,8 \%$ & NS \\
\hline & Industria & $12 \%$ & $11,9 \%$ & $12,5 \%$ & - & $11,4 \%$ & $12,1 \%$ & - \\
\hline & Construcción & $7 \%$ & $5,5 \%$ & $15,6 \%$ & - & $2,8 \%$ & $7,4 \%$ & - \\
\hline & Servicios & $26 \%$ & $25,7 \%$ & $25,0 \%$ & - & $31,4 \%$ & $21,6 \%$ & - \\
\hline Empre & familiar & $87 \%$ & $87,0 \%$ & $90,6 \%$ & NS & $86,7 \%$ & $87,2 \%$ & NS \\
\hline Edad & (promedio) & 56 & 56 & 57 & NS & 56 & 56 & NS \\
\hline & Primario & $10 \%$ & $9,9 \%$ & $12,5 \%$ & NS & $14,3 \%$ & $6,7 \%$ & $(+)^{*}$ \\
\hline Educación & Medio & $52 \%$ & $51,4 \%$ & $56,2 \%$ & - & $52,4 \%$ & $50,6 \%$ & - \\
\hline & Superior & $37 \%$ & $38,7 \%$ & $31,2 \%$ & - & $33,3 \%$ & $42,6 \%$ & - \\
\hline Objetivo & mpresario & $52 \%$ & $51,7 \%$ & $55,5 \%$ & NS & $44,4 \%$ & $57 \%$ & $(+)^{*}$ \\
\hline Fonde & propios & $80 \%$ & $81 \%$ & $72 \%$ & NS & $89,5 \%$ & $74,3 \%$ & $(-)^{* *}$ \\
\hline Créditc & comercial & $62 \%$ & $62 \%$ & $62,50 \%$ & NS & $52,4 \%$ & $68,2 \%$ & $(+)^{* *}$ \\
\hline Crédit & bancario & $38,9 \%$ & $33,2 \%$ & $84,4 \%$ & $(+)^{* * *}$ & $16,2 \%$ & $45,3 \%$ & $(+)^{* * *}$ \\
\hline $\begin{array}{r}\text { Otro finar } \\
\text { ba }\end{array}$ & $\begin{array}{l}\text { iamiento no } \\
\text { cario }\end{array}$ & $14 \%$ & $14,2 \%$ & $9,40 \%$ & NS & $12,4 \%$ & $15,5 \%$ & NS \\
\hline $\begin{array}{r}\text { Exclusic } \\
\text { ba }\end{array}$ & $\begin{array}{l}\text { al crédito } \\
\text { cario }\end{array}$ & $43 \%$ & $48 \%$ & $3,1 \%$ & $(-)^{* * *}$ & $61 \%$ & $38,5 \%$ & $(-)^{* * *}$ \\
\hline Pérdida & e inversión & $37,50 \%$ & $40 \%$ & $22 \%$ & $(-)^{*}$ & $33 \%$ & $44 \%$ & $(+)^{*}$ \\
\hline
\end{tabular}

Nota: NS: variable no significativa; (+): relación positiva con la variable dependiente; (-): relación inversa con la variable dependiente; ${ }^{* *}$ significativa al $99 \%$ de nivel de confianza; ${ }^{* *}$ significativa al nivel de $95 \%$ de confianza; ${ }^{*}$ significativa al nivel de $90 \%$ de confianza.

Fuente: elaboración propia. 
Analizando el grupo de empresas que demandarían un CTS (DP $=1$ ), se observa que en este grupo hay una menor participación de empresas micro, y una mayor participación de empresas pequeñas, respecto al grupo de empresas que no demandarían este instrumento. Por su parte, la antigüedad tiene una relación positiva y significativa, al igual que la forma jurídica, y ello indica que las potenciales demandantes de CTS son más antiguas y se conforman en mayor medida con formas jurídicas que limitan la responsabilidad patrimonial. En cuanto a las características del gerente, se observa que las potenciales demandantes de CTS tienen gerentes o propietarios con mayor nivel de educación y que priorizan los objetivos empresariales sobre objetivos personales en la toma de decisiones de su empresa.

La estructura financiera nos indica que las empresas que potencialmente demandarían un CTS utilizan en menor proporción fondos propios para financiar la actividad, y en mayor proporción crédito comercial y bancario. En cuanto a la variable que representa las necesidades de financiación bancaria en el pasado, denominada exclusión al crédito bancario, se aprecia una relación negativa con las potenciales demandantes. En este sentido, hay una menor proporción de empresas excluidas del sistema financiero dentro de las potenciales demandantes de CTS. Finalmente, registraron tener una mayor proporción de empresas que vivenciaron una pérdida de inversión.

En resumen, se observa que existen varias coincidencias en cuanto a la relación de las variables independientes tanto con la demanda efectiva como con la demanda potencial. Para profundizar el análisis, estudiaremos dicha relación a partir de un análisis multivariado, cuyos resultados se presentan en la siguiente sección.

\section{Resultados del análisis multivariado}

A fin de conocer en qué medida cada una de las variables independientes afectan la probabilidad de demandar efectiva y potencialmente un CTS, y dada la naturaleza dicotómica de las variables dependientes, el modelo por aplicar es un Logit binario. En la tabla 5 se presentan los resultados de estimar la demanda efectiva de CTS, incorporando en el modelo aquellas variables que resultaron ser significativas en la tabla 4. En primer lugar, se presenta el modelo 1, que registra el impacto de la variable de perfil de la empresa sobre la demanda efectiva de CTS (M1). A partir de este modelo, se incorporan las variables de estructura de financiamiento, cuyos resultados se presentan en el modelo 2 (M2); y, por último, aquellas variables que indican que la empresa presenta problemas para acceder al financiamiento externo, modelo 3 (M3). 
En el M1 se observa que la probabilidad de demandar efectivamente un CTS (PrDE) aumenta en un 8,27\% si la empresa es pequeña y un $24,68 \%$ si la empresa es mediana respecto a las microempresas. Dichos resultados indicarían que cuanto más grande es la empresa, mayor es la probabilidad de haber solicitado un CTS.

Se observa además que las empresas con formas jurídicas que limitan responsabilidad patrimonial, como una SA o una SRL, tienen una probabilidad de haber solicitado un CTS un 6,37\% mayor respecto a las incluidas en el régimen de autónomos. En M2 se incorpora la variable de crédito bancario y el modelo mejora su poder explicativo. ${ }^{4} \mathrm{Si}$ bien la forma jurídica deja de ser significativa, se aprecia que la PrDE aumenta en 15,64\% para las empresas que tienen créditos bancarios como fuente de financiamiento habitual, en relación con las que no utilizan este tipo de financiamiento. En el M3 se observa que si la empresa presentó necesidad de crédito bancario insatisfecha en el pasado, es decir, algún nivel de exclusión al crédito bancario, la PrDE disminuye en un $14,98 \%$ en comparación con la PrDE de las empresas no excluidas.

Los resultados respecto a tamaño y relación previa con el sistema bancario coinciden con estudios previos (Briozzo y Vigier, 2009b; Bueso-Merrieam et al., 2016; Butler et al., 2017).

En la tabla 6 se presentan los efectos marginales de demandar potencialmente un CTS. Al igual que en la tabla 5, el M1 es un modelo que registra el impacto de las variables de perfil de la empresa, el M2 incorpora las variables de estructura de financiamiento y el M3 incorpora variables indicativas de problemas para acceder al financiamiento externo. Tanto en M2 como en M3 aumenta el poder explicativo de los modelos respecto a M1.

Con respecto a las características de la empresa, se observa que en los tres modelos el tamaño de la empresa deja de ser una variable significativa, y ello indica que no existen diferencias en la probabilidad de demandar potencialmente un CTS entre las más pequeñas y las más grandes dentro del universo mipyme, a diferencia de lo que sucedía con la demanda efectiva. Además, la antigüedad de la empresa solo es significativa en el M1, y la forma jurídica es significativa en todos los casos, aunque se ve reducido su efecto conforme se incorporan otras variables de interés.

4 Se observa que tanto el peusoR2 como el countR2 aumentan su valor, indicando una mejora en el poder explicativo del modelo en su conjunto. 
Tabla 5.

\begin{tabular}{|c|c|c|c|c|}
\hline \multicolumn{5}{|c|}{ Demanda efectiva de crédito bancario a tasa subsidiada } \\
\hline \multirow{2}{*}{\multicolumn{2}{|c|}{ Variables explicativas }} & \multicolumn{3}{|c|}{ Demanda efectiva (efectos marginales) } \\
\hline & & M1 & M2 & M3 \\
\hline \multirow{2}{*}{ Tamaño } & Pequeña & $0,0827^{* *}$ & 0,0494 & $0,0710^{* *}$ \\
\hline & Mediana & $0,2468^{* * *}$ & $0,1748^{* * *}$ & $0,1723^{* * *}$ \\
\hline \multicolumn{2}{|c|}{ Antigüedad } & $-0,0054$ & $-0,0208$ & $-0,0194$ \\
\hline \multicolumn{2}{|c|}{ Forma jurídica } & $0,0637^{*}$ & 0,0440 & 0,0332 \\
\hline \multicolumn{2}{|c|}{ Crédito bancario } & - & $0,1564^{* * *}$ & - \\
\hline \multicolumn{2}{|c|}{ Exclusión al crédito bancario } & - & - & $-0,1498^{* * *}$ \\
\hline \multicolumn{2}{|c|}{ Pérdida de inversión } & - & - & $-0,0429$ \\
\hline \multicolumn{5}{|c|}{ Estadísticos del modelo } \\
\hline \multicolumn{2}{|c|}{ Log_lik Intercept Only } & $-99,989$ & $-99,989$ & $-99,989$ \\
\hline \multicolumn{2}{|c|}{ Log_lik Full Model } & $-84,589$ & $-76,449$ & $-75,558$ \\
\hline \multicolumn{2}{|c|}{ Wald chi2 } & 25,4 & 43,31 & 30,09 \\
\hline \multicolumn{2}{|c|}{ Prob $>$ chi 2} & 0,0000 & 0,0000 & 0,0000 \\
\hline \multicolumn{2}{|c|}{ Pseudo R2 } & 0,1540 & 0,2354 & 0,2443 \\
\hline \multicolumn{2}{|c|}{ Count R2 } & 0,887 & 0,891 & 0,884 \\
\hline \multicolumn{2}{|c|}{$\mathrm{N}$} & 284 & 284 & 284 \\
\hline
\end{tabular}

Nota: ${ }^{* * *}$ significativa al 99\% de nivel de confianza; ${ }^{* *}$ significativa al nivel de $95 \%$ de confianza; ${ }^{*}$ significativa al nivel de 90\% de confianza independientes; M1: Modelo 1; M2: Modelo 2; M3: Modelo 3.

Fuente: elaboración propia.

En M1, ser una empresa que limita su responsabilidad aumenta la probabilidad de demandar potencialmente un CTS (PrDP) un 19,19\%, y si el propietario-gerente tiene un nivel de educación superior, aumenta un 23,2\%. En M2 se observa que la PrDP aumenta un 15,61\% si tiene una forma jurídica con responsabilidad limitada, respecto a las que no limitan. Por otro lado, si el gerente tiene un nivel de educación medio, la PrDP aumenta un 17,29\%, y un $23,48 \%$ si este tiene un nivel de educación superior, respecto a los que solo alcanzaron el nivel primario. En relación con las fuentes de financiamiento, la PrDP aumenta un 12,07\% si la empresa utiliza crédito comercial y un $19,07 \%$ si utiliza crédito bancario, respecto a las que no se financian con este tipo de instrumentos. Estos resultados muestran que el acceso a otras fuentes de financiamiento externas nuevamente es la principal variable que afecta la demanda potencial de CTS. 
Lilia José Corzo • María Belén Guercio • Hernán Pedro Vigier

En M3, la PrDP disminuye un $12,27 \%$ si la empresa se encuentra excluida al crédito bancario respecto a las empresas que no tienen dicha limitación. Nuevamente, la relación previa del empresario con el sistema bancario afecta su comportamiento respecto a la consideración del uso de un CTS. Mientras que haber registrado una pérdida de inversión aumenta en un 16,23\% la PrDP.

Tabla 6.

Demanda potencial de crédito bancario a tasa subsidiada

\begin{tabular}{|c|c|c|c|c|}
\hline \multirow{2}{*}{\multicolumn{2}{|c|}{ Variables explicativas }} & \multicolumn{3}{|c|}{ Demanda potencial (efectos marginales) } \\
\hline & & \multirow{2}{*}{$\begin{array}{c}\text { M1 } \\
0,1083\end{array}$} & \multirow{2}{*}{$\begin{array}{c}\text { M2 } \\
0,0222\end{array}$} & \multirow{2}{*}{$\begin{array}{c}\text { M3 } \\
0,0743\end{array}$} \\
\hline \multirow{2}{*}{ Tamaño } & Pequeña & & & \\
\hline & Mediana & 0,0779 & $-0,1420$ & $-0,0620$ \\
\hline Antigüedad & & $0,0585^{*}$ & 0,0292 & 0,0330 \\
\hline Forma jurídica & & $0,1919^{* * *}$ & $0,1561^{* *}$ & $0,1444^{* *}$ \\
\hline \multirow{2}{*}{ Educación } & Medio & 0,1555 & $0,1729^{*}$ & $0,1952^{*}$ \\
\hline & Superior & $0,2320^{* *}$ & $0,2348^{* *}$ & $0,2673^{* *}$ \\
\hline \multicolumn{2}{|c|}{ Objetivo empresario } & 0,0511 & 0,0721 & 0,0795 \\
\hline \multicolumn{2}{|c|}{ Fondos propios } & - & $-0,1286$ & $-0,1287$ \\
\hline \multicolumn{2}{|c|}{ Crédito comercial } & - & $0,1207^{* *}$ & $0,1671^{* * *}$ \\
\hline \multicolumn{2}{|c|}{ Crédito bancario } & - & $0,1907^{* * *}$ & - \\
\hline \multicolumn{2}{|c|}{ Exclusión al crédito bancario } & - & - & $-0,1227^{* *}$ \\
\hline \multicolumn{2}{|c|}{ Pérdida de inversión } & - & - & $0,1623^{* * *}$ \\
\hline \multicolumn{2}{|c|}{ Estadísticos del modelo } & - & - & - \\
\hline \multicolumn{2}{|c|}{ Log_lik Intercept Only } & $-158,553$ & $-158,553$ & $-158,553$ \\
\hline \multicolumn{2}{|c|}{ Log_lik Full Model } & $-145,662$ & $-137,929$ & $-135,916$ \\
\hline \multicolumn{2}{|c|}{ Wald chi2 } & 21,39 & 27,06 & 32 \\
\hline \multicolumn{2}{|c|}{ Prob > chi2 } & 0,0032 & 0,0026 & 0,0008 \\
\hline \multicolumn{2}{|c|}{ Pseudo R2 } & 0,0813 & 0,1301 & 0,1428 \\
\hline \multicolumn{2}{|c|}{ Count R2 } & 0,648 & 0,708 & 0,687 \\
\hline \multicolumn{2}{|c|}{$\mathrm{N}$} & 233 & 233 & 233 \\
\hline
\end{tabular}

Nota: ${ }^{* * *}$ variable significativa al $99 \%$ de nivel de confianza; ${ }^{* *}$ significativa al nivel de $95 \%$ de confianza; " significativa al nivel de 90\% de confianza; M1: Modelo 1; M2: Modelo 2; M3: Modelo 3.

Fuente: elaboración propia. 


\section{CONCLUSIONES}

Las mipymes argentinas son foco de políticas públicas diversas desde hace décadas. Se reconoce la importancia de estas empresas para el entramado productivo y las dificultades en el acceso al financiamiento externo desde los distintos sectores gubernamentales que las atienden. Particularmente, el otorgamiento de créditos con tasas subsidiadas es uno de los instrumentos más extendido y utilizado. Sin embargo, el alcance de estas medidas es prácticamente desconocido y los abordajes son parciales.

Este trabajo profundiza el análisis acerca de las características tanto de las empresas que demandaron efectivamente un crédito a tasa subsidiada (CTS), como de aquellas que estarían dispuestas a demandarlo y no lo han hecho. Los modelos de regresión logística construidos permiten abordar conclusiones inferenciales. Tanto para las empresas con demanda efectiva como para las demandantes potenciales, los resultados son concluyentes respecto a la importancia de la relación previa de la empresa con el mercado bancario para determinar el alcance del instrumento. Este resultado surge de considerar tanto el uso frecuente de crédito bancario, así como de haber tenido una necesidad de financiación insatisfecha, medida como la exclusión al crédito bancario en el pasado.

Uno de los resultados más relevantes de este trabajo es que las empresas excluidas al crédito bancario presentan una menor probabilidad de haber demandado o de demandar potencialmente un CTS. Este resultado se puede observar en la elevada proporción de empresas excluidas del crédito bancario que potencialmente demandarían (38,5\%), en relación con las que efectivamente demandaron $(3,1 \%)$. Ello demuestra el bajo alcance de las acciones de política pública, específicamente de los CTS, instrumento que parecería que comparte las mismas dificultades que los instrumentos bancarios tradicionales.

Una de las escasas variables del perfil de la empresa que afecta la probabilidad de haber demandado un CTS es el tamaño. Dicha relación estaría indicando que las empresas más grandes, dentro de las tres categorías de mipymes, son las que demandan efectivamente un CTS; sin embargo, no se mantiene en la demanda potencial. A este resultado hay que sumarle el hecho de que cuando las empresas son micro y pequeñas, el porcentaje de empresas que estarían dispuestas a demandar un CTS es mucho mayor al que efectivamente demandó. Por ello, los nuevos instrumentos de política pública deben enfocarse en las empresas de menor tamaño, las cuales representan un mayor número dentro del agregado mipymes. 
De esta forma, los programas públicos de apoyo financiero mejorarían su alcance y cumplirían el objetivo para el cual fueron creados.

Otras características de la empresa que explican la demanda potencial de CTS son: la forma jurídica, el nivel de educación del gerente, el acceso al crédito comercial y si ha perdido realizar una inversión por falta de fondos. Sobre la forma jurídica, la relación encontrada indicaría que las empresas que limitan responsabilidad generan mayor información contable y escrita para brindar a las instituciones de crédito. Por esta razón, tienen una mayor probabilidad de demandar potencialmente un CTS por contar con recursos para demostrar su capacidad y voluntad de pago. Respecto al nivel de educación del propietario gerente, la relación positiva se puede deber a que las personas más formadas puedan reconocer las ventajas de endeudarse con un CTS, en lugar de otro tipo de instrumento financiero.

En lo concerniente al acceso al financiamiento externo, se encuentra que las empresas que utilizan créditos comerciales presentan una mayor probabilidad de demandar potencialmente un CTS. En este sentido, el resultado podría indicar que este segmento de empresas estaría dispuesto a complementar su financiamiento habitual de crédito comercial con CTS, en tanto podrían encontrar una fuente alternativa de menor costo. Este resultado, en simultáneo con la significatividad de la forma jurídica, muestra que este grupo de empresas con menos opacidad de información serían más propensas a tomar CTS.

Finalmente, la experiencia previa con la pérdida de inversión rentable por falta de fondos aumenta la probabilidad de demandar un CTS. Este hallazgo muestra que, de mejorarse las condiciones de acceso al instrumento de política pública, se alcanzaría a las empresas con mayores dificultades en el uso del crédito.

En resumen, esta investigación permite evaluar un instrumento de política pública muy difundido, demarcar sus limitaciones y resaltar sus aciertos. Si bien la relación previa de la empresa con el mercado bancario es determinante para el acceso CTS, existe una demanda potencial dispuesta a superar ciertas condicionantes del instrumento que posibilitaría la mejora en su alcance.

El estudio de la demanda potencial demostró que el instrumento CTS puede mejorar su alcance y enfocarse en ciertos perfiles de empresas mipymes excluidas. Este perfil coincide con aquellas más formalizadas, con necesidades de fondos externos y con dificultades para acceder al financiamiento bancario tradicional. Son sumamente relevantes los resultados hallados respecto a la demanda potencial y 
su consideración es novedosa. El aporte de este trabajo de investigación puede ser fundamental para el rediseño y la adecuación de los programas de financiamiento para mipymes y explica el bajo alcance de programas anteriores.

\section{AGRADECIMIENTOS}

Los autores agradecen a los evaluadores de la revista Finanzas y Política Económica sus sugerencias para mejorar la calidad del artículo. Este artículo es resultado de un trabajo de investigación tendiente a obtener el grado de doctorado de la autora Lilia Corzo, becaria doctoral cofinanciada por la Comisión de Investigaciones Científicas de la Provincia de Buenos Aires y la Universidad Provincial del Sudoeste.

\section{REFERENCIAS}

1. Agresti, A. (2002). Categorical variable analysis. Nueva Jersey: John Wiley \& Sons https://doi.org/10.1002/0471249688

2. Akerlof, G. A. (1970). The market for "Lemons": Quality uncertainty and the market mechanism. Quarterly Journal of Economics, (84), 488-500 https://doi. org/10.2307/1879431

3. Bebczuk, R. N. (2004). What determines the access to credit by SMEs in Argentina? [Working Paper n. ${ }^{\circ}$ 48]. La Plata: Universidad Nacional de La Plata. www.depeco. econo.unlp.edu.ar

4. Bebczuk, R. N. (2010). Acceso al financiamiento de las pymes en la Argentina: estado de situación y propuestas de política. Santiago de Chile: Cepal. https://doi.org/92-1-321831-1

5. Beck, T. y Demirguc-Kunt, A. (2006). Small and medium-size enterprises: Access to finance as a growth constraint. Journal of Banking and Finance, 30(11), 2931-2943. https://doi.org/10.1016/j.jbankfin.2006.05.009

6. Beck, T., Demirgüç-Kunt, A. y Pería, M. S. M. (2011). Bank financing for SMEs: Evidence across countries and bank ownership types. Journal of Financial Services Research, 39(1-2), 35-54. https://doi.org/10.1007/s10693-010-0085-4

7. Berger, A. N. y Udell, G. F. (1998). The economics of small business finance: The roles of private equity and debt markets in the financial growth cycle. Journal of Banking $\mathcal{E}$ Finance, 22(6-8), 613-673. https://doi.org/10.1016/S0378-4266(98)00038-7

8. Berger, A. N. y Udell, G. F. (2006). A more complete conceptual framework for SME finance. Journal of Banking and Finance, 30 (11), 2945-2966. https://doi.org/10.1016/j. jbankfin.2006.05.0081 
9. Binelli, C. y Maffioli, A. (2007). A micro-econometric analysis of public support to private R\&D in Argentina. International Review of Applied Economics, 21 (3), 339-359. https://doi.org/10.1080/02692170701390320

10. Bougheas, S., Mizen, P. y Yalcin, Y. (2006). Access to external financing: Theory and evidence on the impact of monetary policy and firm-specific characteristics. Journal of Banking and Finance, 30(1), 199-227. https://doi.org/10.1016/j.jbankfin.2005.01.002

11. Briozzo, A. y Cardone-Riportella, C. (2016) Spanish SMEs' subsidized and guaranteed credit during economic crisis: A regional perspective. Regional Studies, 50(3), 496-512. https://doi.org/10.1080/00343404.2014.926318

12. Briozzo, A. y Vigier, H. (2006). La estructura de financiamiento PyME. Una revisión del pasado y presente. https://mpra.ub.uni-muenchen.de/id/eprint/5894

13. Briozzo, A. y Vigier, H. (2009a). A demand-side approach to SMEs' capital structure: Evidence from Argentina. Journal of Business and Entrepreneurship, 21 (1), 30-56.

14. Briozzo, A. y Vigier, H. (2009b). La demanda de créditos promocionales en las pequeñas empresas argentinas. XXIX Jornadas Nacionales de Administración Financiera. Córdoba, Argentina. http://repositoriodigital.uns.edu.ar/handle/123456789/4304

15. Bueso-Merriam, J., Demichelis, F., Fernández Díez, M. C., Giuliodori, D., Rodríguez, A. y Stucchi, R. (2016). El impacto del Programa de Crédito para el Desarrollo de la Producción y el Empleo en la Provincia de San Juan [Documento para discusión n. ${ }^{\circ}$ IDB-DP-485]. Washington: BID.

16. Butler, I., Guiñazu, S., Giuliodori, D., Martínez Correa, J., Rodríguez, A. y Tacsir, E. (2017). Programas de financiamiento productivo a PyMES, acceso al crédito y desempeño de las firmas: evidencia de Argentina (n. ${ }^{\circ}$ 2018/05). http://scioteca.caf.com/bitstream/ handle/123456789/1151/programas-de-financiamiento-productivo-a-pymes-acceso-al-credito-y-desempeno-de-las-firmas-evidencia-de-argentina \%28002\%29. pdf? sequence $=5$ yisAllowed $=\mathrm{y}$

17. Castillo, V., Figal Garone, L., Maffioli, A. y Ohaco, M. (2016). Asistencias técnicas y competitividad de las PyMEs: Evidencia para Argentina. Washington: Inter-American Development Bank (IDB). https://doi.org/10.18235/0000568

18. Castillo, V., Maffioli, A., Monsalvo, A. P., Rojo, S. y Stucchi, R. (2010). Can SME Policies Improve Firm Performance? Evidence from an Impact Evaluation in Argentina [OVE Working Papers 0710]. Inter-American Development Bank, Office of Evaluation and Oversight (OVE),

19. Castillo, V., Maffioli, A., Rojo, S. y Stucchi, R. (2011). Innovation Policy and Employment: Evidence from an Impact Evaluation in Argentina. Inter-American Development Bank Science and Technology Division, Social Sector. TECHNICAL NOTES, IDBTN-341. https://publications.iadb.org/en/innovation-policy-and-employment -evidence-impact-evaluation-argentina

20. Castillo, V., Maffioli, A., Rojo, S. y Stucchi, R. (2014). The effect of innovation policy on SMEs' employment and wages in Argentina. Small Business Economics, 42 (2), 387 406. https://doi.org/10.1007/s11187-013-9485-9 
21. Chudnovsky, D., López, A., Rossi, M. y Ubfal, D. (2006). Evaluating a Program of Public Funding of Private Innovation Activities. An Econometric Study of FONTAR in Argentina. OVE Working Papers, Inter-American Development Bank, Office of Evaluation and Oversight (OVE) 1606. https://www.researchgate.net/profile/Andres_Lopez18/ publication/5106181_Evaluating_A_Program_Of_Public_Funding_Of_Private_ Innovation_Activities_An_Econometric_Study_Of_FONTAR_In_Argentina/ links/0f317534ef9961a3eb000000.pdf

22. Cole, R. A., Goldberg, L. G. y White, L. J. (2004). Cookie Cutter vs. Character: The Micro Structure of Small Business Lending by Large and Small Banks. Journal of Financial and Quantitative Analysis, 39(2), 227. http://journals.cambridge.org/ abstract_S0022109000003057

23. Cole, R. y Dietrich, A. (2012). SME credit availability around the world: Evidence from the World Bank's Enterprise Survey. Paper presented at the 2013 Annual Meetings of the Southern Finance Association in Charleston SC USA held Nov. http://dx.doi. org/10.2139/ssrn.2043624

24. Cole, R. y Sokolyk, T. (2016). Who needs credit and who gets credit? Evidence from the surveys of small business finances. Journal of Financial Stability, (24), 40-60. https:// doi.org/10.1016/j.jfs.2016.04.002

25. Cowling, M., Liu, W., Minniti, M. y Zhang, N. (2016). UK credit and discouragement during the GFC. Small Business Economics, 47(4), 1049-1074. http://dx.doi.org/10.1108/ BIJ-10-2012-0068

26. Cravo, T. A. y Piza, C. (2016). The Impact of Business Support Services for Small and Medium Enterprises on Firm Performance in Low- and Middle-Income Countries. A Meta-Analysis. IDB Working Paper Series № IDB-WP-709. https://publications.iadb. org/publications/english/document/The-Impact-of-Business-Support-Services-forSmall-and-Medium-Enterprises-on-Firm-Performance-in-Low--and-Middle--IncomeCountries-A-Meta-Analysis.pdf

27. De La Torre, A., Martínez Pería, M. S. y Schmukler, S. L. (2010). Bank involvement with SMEs: Beyond relationship lending. Journal of Banking y Finance Journal, (34), 2280-2293. http://dx.doi.org/10.1016/j.jbankfin.2010.02.014

28. Dong, Y. y Men, C. (2014). SME Financing in Emerging Markets: Firm Characteristics, Banking Structure and Institutions. Emerging Markets Finance and Trade, 50(1), 120149. http://dx.doi.org/10.2753/REE1540-496X500107

29. Ferraro, C. y Stumpo, G. (2010). Políticas de apoyo a las pymes en América Latina. Entre avances innovadores y desafíos institucionales. Santiago de Chile: Comisión Económica para América Latina y el Caribe (CEPAL).

30. Forte, D., Barros, L. A. y Nakamura, W. T. (2013). Determinants of the capital structure of small and medium sized Brazilian enterprises. BAR - Brazilian Administration Review, 10(3), 347-369. https://doi.org/10.1590/S1807-76922013000300007

31. Freel, M., Carter, S., Tagg, S. y Mason, C. (2012). The latent demand for bank debt: Characterizing "discouraged borrowers". Small business economics, 38(4), 399-418.

32. Goldstein, E. (2011). El crédito a las pymes en la Argentina: Evolución reciente y estudio de un caso innovador (Documento de proyecto). Santiago de Chile: Comisión 
Económica para América Latina y el Caribe (CEPAL). http://archivo.cepal.org/ pdfs/2011/S2011062.pdf

33. Gregory, B. T., Rutherford, M. W., Oswald, S. y Gardiner, L. (2005). An empirical investigation of the Growth Cycle Theory of small firm financing. Journal of Small Business Management, 43(4), 382-392.

34. Grimm, M. y Paffhausen, A. L. (2015). Do interventions targeted at micro-entrepreneurs and small and medium-sized firms create jobs? A systematic review of the evidence for low and middle income countries. Labour Economics, (32), 67-85.

35. Guercio, M. B., Vigier, H. P. y Briozzo, A. E. (2014). The legal form and access to financing for industrial SMEs. DYNA Management, 2(1), 1-8. http://dx.doi.org/10.6036/ MN6946

36. Guercio, M. B., Oliveras, G., Vigier, H. P. y Briozzo, A. E. (2015). Financiamiento externo desde una perspectiva de género. Revista Venezolana de Gerencia, 20(71), 440-454. https://doi.org/10.31876/revista.v20i70.20002

37. Heredia Rodríguez L. y Sánchez Macías J. I. (2016). Evolución de las políticas públicas de fomento a las pymes en la Comunidad Andina de Naciones y la Unión Europea: un análisis comparativo. Revista Finanzas y Política Económica, 8(2), 221-249. DOI: 10.14718/revfinanzpolitecon.2016.8.2.2

38. Jensen, M. C. y Meckling, W. H. (1976). Theory of the firm: Managerial behavior, agency costs and ownership structure. Journal of Financial Economics, 3(4), 305-360.

39. Kersten, R., Harms, J., Liket, K. y Maas, K. (2017). Small Firms, large Impact? A systematic review of the SME Finance Literature. World Development, (97), 330-348. https://doi.org/10.1016/J.WORLDDEV.2017.04.012

40. Kon, Y. y Storey, D. J. (2003). A theory of discouraged borrowers. Small Business Economics, 21 (1), 37-49.

41. Kulfas, M. (2009). Las PYMES argentinas en el escenario post convertibilidad: políticas públicas, situación y perspectivas. Documento de proyecto. Buenos Aires: CEPAL. http:// repository.eclac.org/handle/11362/3718

42. Kumar, A. y Francisco, M. (2005). Enterprise size, financing patterns, and credit constrains in Brazil. Analysis of data from the investment climate assessment survey. The World Bank Working Paper, (49), 1-55. https://doi.org/10.1596/0-8213-6129-5

43. Lavarello, P. y Sarabia, M. (2015). La política industrial en la Argentina durante la década de 2000 (Estudios y perspectivas n. ${ }^{\circ}$ 45). Santiago de Chile: Comisión Económica para América Latina y el Caribe (CEPAL). repositorio.cepal.org/bitstream/handle/11362/ 39886/1/S1600018_es.pdf

44. Liu, Q. y Tian, G. G. (2009). Leverage ratio and determinants of capital structure in SMEs: evidence from China. $22^{\text {nd }}$ Proceedings of the Australasian Finance and Banking Conference, Sydney, Australia. http://dx.doi.org/10.2139/ssrn.1460375

45. Llisterri, J., Vivanco, F. y Ariano, M. (2002). Acceso de las pequeñas y medianas empresas al financiamiento. Informe de Trabajo. Washington, DC: Banco Interamericano de Desarrollo. 
46. Martincus, C. V. y Carballo, J. (2010). Beyond The Average Effects: The Distributional Impacts of Export Promotion Programs in Developing Countries. Journal of Development Economics, (92), 201-214. https://publications.iadb.org/es/publicacion/10664/ beyond-average-effects-distributional-impacts-export-promotion-programs

47. Martincus, C. V., Carballo, J. y Garcia, P. M. (2014). Public Programmes to Promote Firm's Exports in Developing Countries: Are There Heterogeneous Effects By Size Categories? Applied Economics, (44), 471-491.

48. Miller, M. H. (1977). Debt and Taxes. Journal of Finance, 32 (2), 261-275. https://doi. org/10.2307/2326758

49. Ministerio de Economía y Finanzas Públicas (2011). Instrumentos para el desarrollo productivo en la Argentina: análisis de las políticas de incentivo a la producción (1. ${ }^{\mathrm{a}}$ ed.). Buenos Aires. https://www.economia.gob.ar/ baseinstrumentos/documentos.html

50. Ministerio de Hacienda (2019). Política para el acceso al crédito productivo. https://www. argentina.gob.ar/sites/default/files/3.1_pacp-politica_de_acceso_al_credito_productivo_vf_0.pdf

51. Modigliani, F. y Miller M. (1958). The cost of capital, corporation finance and the theory of investment. The American Economic Review, (48), 261-297.

52. Modigliani, F. y Miller M. (1963). Corporate income taxes and the cost of capital: a correction. The American Economic Review, (53), 433-443. https://doi. org/10.1213/00000539-197405000-00034

53. Myers, S. C. (1984). The capital structure puzzle. Journal of Finance, 39(3), 574-592. https://doi.org/10.2307/2327916

54. Myers, S. C. y Majluf, N. S. (1984). Corporate financing and investment decisions when firms have information that investors do not have, Journal of Financial Economics, 13(2), 187-221.

55. Petersen, M. y Rajan, R. (1994). The benefits of lending relationships: Evidence from small business data. Journal of Finance, 49(1), 3-37. https://doi.org/10.1111/j.1540-6261.1994. tb04418.x

56. Romano, C. A., Tanewski, G. A. y Smyrnios, K. X. (2001). Capital structure decision making: A model for family business. Journal of Business Venturing, 16(3), 285-310. https://doi.org/10.1016/S0883-9026(99)00053-1

57. Rostamkalaei, A. (2017). Discouraged borrowers aftermath of financial crisis: A UK study. Journal of Small Business and Enterprise Development, 24(2), 394-410. http:// dx.doi.org/10.1108/JSBED-09-2016-0137

58. Ruffo, H., Butler, I., Galassi, G. y González, G. (2012). Impacto de las políticas de fomento al emprendedorismo: el caso de "Buenos Aires emprende". http://scioteca.caf.com/ handle/123456789/241

59. Sanguinetti, P. (2005). Innovation and RyD Expenditures in Argentina: Evidence from a firm level survey. http://citeseerx.ist.psu.edu/viewdoc/download? doi=10.1.1.613.1353 $\&$ rep $=$ rep $1 \&$ type $=$ pdf 
60. Steindl, J. (1945). Small and big business. Economic problem of the size of the firms. Oxford: Basil Blackweel.

61. Stiglitz, J. E. (1993). The role of the state in the financial Markets. The World Bank Economic Review, 7(1), 19-52. https://doi.org/10.1093/wber/7.suppl_1.19

62. Stiglitz, J. E. y Weiss, A. (1981). Credit Rationing in Markets with Imperfect Information. The American Economic Review, 71(3), 393-410.

63. Storey, D. J. (1994).Understanding the small business sector. Londres: Routledge.

64. UIA (2018). Los instrumentos de Financiamiento PyMI como un motor para potenciar el desarrollo. Departamento PyMI y Desarrollo Regional de la Unión Industrial Argentina. https://uia.org.ar/pymi-desarrollo-regional/2674/agenda-pymi-financia miento-productivo/

65. Vigier, H., Briozzo, A. y Guercio, M. B (2014). Grandes desafíos para pequeñas empresas: informalidad, financiamiento e información: el caso de las PyMEs de Bahía Blanca. Bahía Blanca: Editorial de la Universidad Nacional del Sur.

66. Wang, Y. (2016). What are the biggest obstacles to growth of SMEs in developing countries? - An empirical evidence from an enterprise survey. Borsa Istanbul Review, 16(3), 167-176. https://doi.org/10.1016/j.bir.2016.06.001

67. Zoppa, A. y McMahon, R. G. P. (2002). Pecking Order Theory and the Financial Structure of Manufacturing SMEs from Australia's Business Longitudinal Survey. Small Enterprise Research, 10(2), 23-41. 